\title{
Integer Sequences Having Prescribed Quadratic Character
}

\author{
By D. H. Lehmer, Emma Lehmer and Daniel Shanks
}

\begin{abstract}
For the odd primes $p_{1}=3, p_{2}=5, \cdots$, we determine integer sequences $N_{p}$ such that the Legendre symbol $\left(N / p_{i}\right)= \pm 1$ for all $p_{i} \leqq p$ for a prescribed array of signs \pm 1 ; (i.e., for a prescribed quadratic character). We examine six quadratic characters having special interest and applications. We present tables of these $N_{p}$ and examine some applications, particularly to questions concerning extreme values for the smallest primitive root (of a prime $N$ ), the class number of the quadratic field $R(\sqrt{ }-N)$, the real Dirichlet $L$ functions, and quadratic character sums.
\end{abstract}

Introduction. Let $p_{1}, p_{2}, \ldots, p_{m}$ be a set of odd primes and let $\varepsilon_{1}, \varepsilon_{2}, \ldots, \varepsilon_{m}$ be a sequence with $\varepsilon^{2}=1$. The problem considered here is that of finding an integer $N$ such that

$$
\left(N / p_{i}\right)=\varepsilon_{i}, \quad(i=1(1) m),
$$

where the symbol is that of Legendre. In other words we are looking for a positive integer $N$ whose quadratic character with respect to each of the given $p_{i}$ is specified. This is a special case of a more general problem of Kummer in which the $\varepsilon$ 's are $k$ th roots of unity and the symbols are $k$ th power characters. This problem has infinitely many solutions for every $k$, cf. Mills [1].

For $k=2$ the infinitude of solutions follows from the law of quadratic reciprocity, since $N$ lies in an arithmetical progression of difference $4 p_{i}$ for each of the $m$ values of $i$, and hence there exists an arithmetical progression of difference $4 p_{1} p_{2} \ldots p_{m}$ every term of which is a desired number $N$. This argument can also be used to obtain the asymptotic density of the N's and even the density of prime values of $N$, but it fails to give any information about the smallest positive value of $N$.

The problem of finding the values of $N$ in natural order is solved automatically by the Delay Line Sieve, DLS 127 [2] provided the $p_{i} \leqq 127$. In what follows we consider six problems of special interest which have applications to other branches of the theory of numbers. In these problems $p_{1}=3, p_{2}=5, \ldots, p_{i}$ is the $i$ th odd prime, the $\varepsilon$ 's form a simple pattern, and $N$ is usually specified modulo 8 .

We present tables of these integer sequences $N$ for the several problems considered, and examine some of their applications, particularly to questions concerning primitive roots, class numbers, Dirichlet $L$ functions, and quadratic character sums. For example, we show that if any algebraic field $R(\sqrt{ }-\Delta)$ of class number 3 exists besides the known examples, then $\Delta>1.4 \cdot 10^{12}$.

Received August 22, 1969.

AMS Subject Classifications. Primary 1016, 1060; Secondary 1041, 1064, 1066.

Key Words and Phrases. Quadratic character, sieves, primitive roots, class number, Dirichlet $L$ functions, quadratic character sums, pseudo-squares. 
Problem I. Find $N \equiv 1(\bmod 8)$ with $\varepsilon_{i}=1$ for all odd $p_{i} \leqq p$ in (1). A solution $N_{p}$ of this problem is a quadratic residue $(\neq 0)$ of all primes $p_{i} \leqq p$ and hence every odd square satisfies the conditions of the problem. We shall be interested here in solutions which are not perfect squares and which have been called pseudo-squares.

Marshall Hall [3] has shown how to use these numbers for a test for primality. Cobham [4] pointed out that the pseudo-squares afford a cheap way of deciding whether a given number is a perfect square or not. Kraitchik [5] listed the least pseudo-square for $p \leqq 47$, and Lehmer [6] and [7] extended this list to $p \leqq 61$, and $p \leqq 79$, respectively. Using the DLS 127 this table was recently extended to $p \leqq 127$. For completeness we give the least pseudo-square for $3 \leqq p \leqq 127$ in Table $\mathrm{I}$.

TABLE I

Table of Pseudo-Squares

\begin{tabular}{|c|c|c|c|}
\hline$p$ & Least Solution & $\begin{array}{l}\text { Least Prime } \\
\text { Solution }\end{array}$ & $\begin{array}{l}\text { Least } \\
\text { Prim. } \\
\text { Root }\end{array}$ \\
\hline 3 & 73 & 73 & 5 \\
\hline 5 & 241 & 241 & 7 \\
\hline 7 & 1009 & 1009 & 11 \\
\hline 11 & $2641=19 \cdot 139$ & 2689 & 19 \\
\hline 13 & 8089 & 8089 & 17 \\
\hline 17 & $18001=47 \cdot 383$ & 33049 & 29 \\
\hline 19 & 53881 & 53881 & 31 \\
\hline 23 & 87481 & 87481 & 29 \\
\hline 29 & $117049=67 \cdot 1747$ & 483289 & 31 \\
\hline 31 & 515761 & 515761 & 37 \\
\hline 37 & 1083289 & 1083289 & 41 \\
\hline 41 & $3206641=643 \cdot 4987$ & 3818929 & 53 \\
\hline 43 & 3818929 & 3818929 & 53 \\
\hline 47 & 9257329 & 9257329 & 53 \\
\hline 53 & 22000801 & 22000801 & 59 \\
\hline 59,61 & 48473881 & 48473881 & 97 \\
\hline 67 & 175244281 & 175244281 & 79 \\
\hline 71,73 & 427733329 & 427733329 & 83 \\
\hline 79 & 898716289 & 898716289 & 101 \\
\hline $83,89,97$ & $2805544681=127 \cdot 859 \cdot 25717$ & Unknown & \\
\hline 101 & $10310263441=4007 \cdot 2573063$ & Unknown & \\
\hline 103 & 23616331489 & 23616331489 & 107 \\
\hline 107,109 & $85157610409=397 \cdot 214502797$ & Unknown & \\
\hline 113,127 & 196265095009 & 196265095009 & 131 \\
\hline
\end{tabular}

The difficulty of this problem is the necessity of eliminating the perfect squares which, to start with, completely upset the expected asymptotic density of the solutions, which is

$$
\Delta_{m}=\frac{1}{8} \prod_{i=1}^{m} \frac{p_{i}-1}{2 p_{i}}
$$


To overcome this difficulty we exploit the capability of the DLS 127 of counting the number of its solutions without actually putting them out. It is clear that the number of unwanted perfect squares $\leqq X$ is exactly the number $\phi\left(P_{m}, X^{1 / 2}\right)$ of numbers prime to $P_{m}=p_{1} p_{2} \ldots p_{m}$ and $\leqq X^{1 / 2}$. This, in turn, is the number of solutions $x \leqq X^{1 / 2}$ given by the Sieve of the trivial Diophantine equation $x y=1$. These two problems were run alternately, using a logarithmic search procedure until the extra nonsquare solution was located and verified.

The deviations from the probabilistic estimate (2) caused by the squares is very marked. While odd perfect squares automatically satisfy $N \equiv 1(\bmod 8)$ and $\varepsilon_{i}=1$, and there are therefore many more solutions of (1) than is indicated by (2), the number of pseudo-square solutions is substantially smaller. For example, for $m=22, p_{i} \leqq$ $p_{m}=83$, there are 168091 solutions of (1) less than $1308943^{2}=1713331777249$. But 161409 of these are squares, and only 6682 pseudo-square solutions occur. The number of solutions predicted by (2) is 12554 .

Western and Miller [8] tabulate the least prime solution $N_{p}$ for $p \leqq 53$. By the law of quadratic reciprocity this is equivalent to finding the least prime $N_{p}$ whose least quadratic nonresidue exceeds $p$. This insures that every prime $<N_{p}$ has a quadratic nonresidue less than $p$ and that there exist primes with arbitrarily large least primitive roots. In Table I, the least prime solution $N_{p}$ and its least primitive root is also listed.

Western and Miller also give a companion table of least negative prime solutions. From our point of view this corresponds to the following problem.

Problem II. Find $N \equiv-1(\bmod 8)$ with $\varepsilon_{i}=\left(-1 / p_{i}\right)$ for all $p_{i} \leqq p$. The negatives $-N_{p}$ of the solutions of this problem are quadratic residues $(\neq 0)$ of all primes $p_{i} \leqq p$ and hence can be thought of as negative pseudo-squares.

This time there is no direct interference from actual squares and one may expect a more predictable distribution. The following short table is for $p_{m}=53, m=15$, $\Delta_{m}=1.03829 \cdot 10^{-6}$.

$\begin{array}{rccc}\text { Limit } \cdot 10^{-6} & \text { No. of Sol. } & \text { Exp. No. } & \text { No. of Sol./Exp. No. } \\ 275 & 150 & 286 & .524 \\ 324 & 200 & 336 & .595 \\ 466 & 300 & 484 & .620 \\ 725 & 500 & 753 & .664 \\ 1297 & 1000 & 1347 & .742 \\ 2720 & 2500 & 2824 & .885 \\ 19617 & 18560 & 20368 & .911 \\ 28925 & 27950 & 30033 & .931 \\ 81324 & 80654 & 84438 & .955 \\ 97900 & 97463 & 101649 & .959 \\ 117000 & 116780 & 121480 & .961\end{array}$

If Problems I and II are thought of as a single problem, one can conjecture that the density of negative pseudo-squares approaches the expected value as the limit $\rightarrow \infty$ and the influence of the perfect squares recedes. 
TABLE II

Negative Squares

$\begin{array}{lr}p & \text { Least Solution } \\ 3 & 23 \\ 5 & 71 \\ 7 & 311 \\ 11 & 479 \\ 13 & 1559 \\ 17 & 5711 \\ 19 & 10559 \\ 23 & 18191 \\ 29 & 31391 \\ 31 & 307271=109 \cdot 2819 \\ 37,41 & 366791 \\ 43 & 2155919=59 \cdot 36541 \\ 47 & 2155919 \\ 53 & 2155919 \\ 59 & 6077111=1039 \cdot 5849 \\ 61 & 6077111 \\ 67 & 98538359=79 \cdot 1247321 \\ 71 & 120293879 \\ 73,79 & 131486759 \\ 83 & 508095719=367 \cdot 547 \cdot 2531 \\ 89 & 2570169839=439 \cdot 5854601 \\ 97 & 2570169839 \\ 101,103 & 2570169839 \\ 107 & 2570169839 \\ 109 & 2570169839 \\ 113,127 & 328878692999 \\ 131 & 513928659191\end{array}$

\begin{tabular}{|c|c|}
\hline Least Prime & $\begin{array}{l}\text { Least } \\
\text { Prim. }\end{array}$ \\
\hline Solution & Root \\
\hline 23 & 5 \\
\hline 71 & 7 \\
\hline 311 & 17 \\
\hline 479 & 13 \\
\hline 1559 & 19 \\
\hline 5711 & 19 \\
\hline 10559 & 23 \\
\hline 18191 & 29 \\
\hline 31391 & 31 \\
\hline 366791 & 43 \\
\hline 366791 & 43 \\
\hline 4080359 & 47 \\
\hline 12537719 & 53 \\
\hline 30706079 & 59 \\
\hline 36415991 & 67 \\
\hline 82636319 & 67 \\
\hline 120293879 & 73 \\
\hline 120293879 & 73 \\
\hline 131486759 & 83 \\
\hline 2929911599 & 97 \\
\hline 2929911599 & 97 \\
\hline 7979490791 & 109 \\
\hline 33857579279 & 107 \\
\hline 89206899239 & 109 \\
\hline 121560956039 & 113 \\
\hline 328878692999 & 131 \\
\hline 513928659191 & 139 \\
\hline
\end{tabular}

The least solution of Problem II for each $3 \leqq p \leqq 131$, and the least prime solution, in case the least solution is composite, is given in Table II together, again, with the primitive roots.

The negative squares have the property that the corresponding quadratic imaginary fields $R(\sqrt{ }-N)$ have exceptionally large class numbers relative to $\sqrt{ } N$, and exceptionally large real Dirichlet $L$ functions at argument 1 :

$$
L(1, \chi)=\sum_{n=1}^{\infty}\left(\frac{-N}{n}\right) \frac{1}{n} .
$$

Here, $(-N / n)$ is the Kronecker symbol. A reflection of this property is that for all $-N_{p}$ listed in Table II with $p>11$ there exist reduced, binary quadratic forms

$$
(A, B, C)=A u^{2}+B u v+C v^{2}
$$

of discriminant $-N_{p_{m}}=B^{2}-4 A C$ for every $A=1,2,3, \ldots$ less than $p_{m+1}$. For example, for $N_{97}=7979490791$, we have 
INTEGER SEQUENCES

TABLE IIa

Negative Squares

\begin{tabular}{|c|c|c|c|c|}
\hline$p$ & $h\left(-N_{p}\right)$ & $L(1, \chi)$ & $h\left(-N_{p}\right)$ & $L(1, \chi)$ \\
\hline 3 & & & 3 & 1.96520 \\
\hline 5 & & & 7 & 2.60987 \\
\hline 7 & & & 19 & 3.38472 \\
\hline 11 & & & 25 & 3.58858 \\
\hline 13 & & & 51 & 4.05786 \\
\hline 17 & & & 109 & 4.53127 \\
\hline 19 & & & 153 & 4.67767 \\
\hline 23 & & & 213 & 4.96137 \\
\hline 29 & & & 289 & 5.12442 \\
\hline 31 & 992 & 5.62213 & 1121 & 5.81495 \\
\hline 37,41 & & & 1121 & 5.81495 \\
\hline 43 & 2968 & 6.35035 & 3997 & 6.21634 \\
\hline 47 & 2968 & 6.35035 & 7457 & 6.61614 \\
\hline 53 & 2968 & 6.35035 & 12017 & 6.81293 \\
\hline 59 & 5092 & 6.48918 & 12719 & 6.62151 \\
\hline 61 & 5092 & 6.48918 & 20299 & 7.01518 \\
\hline 67 & 21934 & 6.94169 & 24503 & 7.01855 \\
\hline 71 & & & 24503 & 7.01855 \\
\hline 73,79 & & & 25817 & 7.07318 \\
\hline 83 & 51460 & 7.17211 & 128755 & 7.47286 \\
\hline 89 & 122106 & 7.56669 & 128755 & 7.47286 \\
\hline 97 & 122106 & 7.56669 & 219207 & 7.70933 \\
\hline 101,103 & 122106 & 7.56669 & 456929 & 7.80137 \\
\hline 107 & 122106 & 7.56669 & 761619 & 8.01103 \\
\hline 109 & 122106 & 7.56669 & 883537 & 7.96118 \\
\hline 113,127 & & & 1499699 & 8.21554 \\
\hline 131 & & & 1870227 & 8.19583 \\
\hline
\end{tabular}

$(1,1,1994872698), \quad(2, \pm 1,997436349), \quad(3, \pm 1,664957566)$,

$(4, \pm 3,498718175), \quad(5, \pm 3,398974540), \quad(6, \pm 1,332478783)$,

$(6, \pm 5,332478784), \ldots \ldots \ldots \ldots \ldots . . . . . .60, \pm 53,19948734)$.

Similarly, for this discriminant, the series (3) begins as the harmonic series

$$
1+\frac{1}{2}+\frac{1}{3}+\cdots+\frac{1}{100}-\frac{1}{101}
$$

with the first hundred terms positive.

We list in Table IIa these class numbers $h\left(-N_{p}\right)$ and these functions $L(1, \chi)$ for both prime and composite $N_{p}$. The composite cases (with even class numbers) are on the left, as in Table II. These numbers, which are related by 


$$
L(1, \chi)=\pi h(-N) / \sqrt{ } N,
$$

were computed by the method described in [9].

Returning briefly to Problem I, we note that a Table Ia would begin:

$\begin{array}{rcccc}p & h(N) & L(1, \chi) & h(N) & L(1, \chi) \\ 3 & & & 1 & 1.79464 \\ 5 & & & 1 & 2.41835 \\ 7 & & & 7 & 3.07844 \\ 11 & 2 & 3.50737 & 1 & 3.48451^{1} \\ 13 & & & 1 & 3.96332\end{array}$

We have not completed this table as the functions are much more difficult to compute for large positive discriminants. But two comments are in order. First, while these $L(1, \chi)$ tend to be large, no particular correlation is indicated for the corresponding class numbers. Instead of (4) one has

$$
L(1, \chi)=\sum_{n=1}^{\infty}\left(\frac{N}{n}\right) \frac{1}{n}=\frac{\ln (u+v \sqrt{ } N) h(N)}{\sqrt{ } N}
$$

where $u^{2}-N v^{2}=1$ is the smallest Pell solution. Usually, $h(N)$ is quite small, and the largeness of $L(1, \chi)$ is reflected, instead, in an exceptionally long period for the regular continued fraction for $\sqrt{ } N$. (It is this that makes the computation difficult.) Secondly, while the $L(1, \chi)$ are relatively large, they are not as large as could be expected by a simple probabilistic estimate. This, again, reflects the peculiarities in the distribution of the pseudo-squares discussed above. While the phenomenon is of interest, we will not pursue it here.

The problem which can be thought of as complementary to Problem II is as follows.

Problem III. Find $N \equiv 3(\bmod 8)$ with $\varepsilon_{i}=-\left(-1 / p_{i}\right)$ for $p_{i} \leqq p$. The negatives $-N_{p}$ of the solutions of this problem are quadratic nonresidues $(\neq 0)$ of all the primes $p_{i} \leqq p$. Such numbers were first considered by Euler in connection with quadratic functions whose values have a high density of primes. In fact, the polynomial

$$
x^{2}+x+(N+1) / 4
$$

of discriminant $-N$ will not be divisible by 2 , or any of the specified primes. Euler hit upon the remarkable $N_{37}=163$, which led to the well-known polynomial $x^{2}+x+41$. Other polynomials of this sort were proposed by Beeger, Poletti, and others [10]-[11]. See also [14].

Since it was established by Stark [12] that 163 is the largest number $N$ with a class number $h(-N)=1$, we know that there does not exist another value of $N$ for which the polynomial (6) is a prime for all values of $x<(N+1) / 4$. Nonetheless, further solutions of Problem III beyond $N_{37}=163$ do provide examples in which the class number $h(-N)$ and $L$ function (3) are apt to be unusually small, while the quadratic polynomial formula (6) possesses an unusually high density of primes. The first two properties are especially obvious when we rewrite (3) in product form:

$$
L(1, \chi)=\frac{\pi h(-N)}{\sqrt{ } N}=\prod_{p=2}^{\infty} \frac{p}{p-(-N / p)}
$$


since we now have $(-N / p)=-1$ for all small $p$, and that minimizes all of the corresponding factors.

Least solutions $N_{p}$ and least prime solutions $N_{p}^{\prime}$ for $3 \leqq p \leqq 163$ are given in Table III. Table IIIa gives the class numbers and $L$ functions. Table III extends an earlier table to $p=107$, by Lehmer [13]. Mohan Lal [14] has also computed the $h\left(-N_{p}\right)$ through $p=107$, and in [14] he, and one of us, discuss some other aspects of this problem.

The decrease of $L(1, \chi)$ with $p$ is, of course, not monotonic. The Legendre symbols beyond $(N \mid p)$ remain unspecified, and if, in these first solutions, these following symbols have an early preponderance of values $\left(-N \mid p_{i}\right)=-1$, as in $N_{127}$, the $L(1, \chi)$ is especially small. Contrarywise, as in $N_{139}^{\prime}$, the $L(1, \chi)$ is "rather poor".

Such small values for $L(1, \chi)$ relate to an investigation of Chowla, Ayoub and Walum [15]. It is known that $h(-q)$ for primes $q \equiv 3(\bmod 4)$ can also be obtained from the sums

$$
S_{1}(q)=\sum_{v=1}^{q-1} v\left(\frac{v}{q}\right)=-q h(-q)
$$

or from

$$
S_{2}(q)=\sum_{v=1}^{q-1} v^{2}\left(\frac{v}{q}\right)=-q^{2} h(-q)
$$

and these quadratic character sums are therefore, of necessity, negative. But in [15] it is proven that

$$
S_{3}(q)=\sum_{v=1}^{q-1} v^{3}\left(\frac{v}{q}\right)
$$

will be positive for infinitely many primes $q$.

To obtain a positive $S_{3}(q)$ it would suffice if

$$
L(1, \chi)<\frac{\zeta(6)}{4 \zeta(2) \zeta(3)}=0.12863,
$$

but that is not easy to attain. No entry in Table IIIa is that small, or even close. For all $q=N_{p}^{\prime}$ listed, we have $S_{3}(q)<0$; e.g., $S_{3}(163)=-2066677=-12679 \cdot 163$.

As was indicated, there is no reason for the first solution $N_{p}^{\prime}$ to be especially good, in this respect, and we have also examined some subsequent solutions. The best prime $q$ presently known to us has

$$
h(-85702502803)=16259 \text { with } L(1, \chi)=0.17448 .
$$

This is a subsequent solution for $p=107$. The smallest $L(1, \chi)$ presently known to us for negative discriminants is that of a large, composite solution for $p=149$ :

$$
N=84148631888752647283=6079 \cdot 30469 \cdot 132137 \cdot 3438209
$$

has

$$
h(-N)=496652272 \quad \text { and } \quad L(1, \chi)=0.17009 .
$$




\section{TABLE III}

$$
\left(\frac{N}{p_{i}}\right)=-\left(\frac{-1}{p_{i}}\right) \text { for all } p_{i} \leqq p, \quad N=8 x+3
$$

\begin{tabular}{|c|c|c|}
\hline$p$ & Least Solution $N_{p}$ & Least Prime Solution $N_{p}^{\prime}$ \\
\hline 3 & 19 & 19 \\
\hline 5,7 & 43 & 43 \\
\hline 11,13 & 67 & 67 \\
\hline $17, \ldots, 37$ & 163 & 163 \\
\hline 41 & $77683=131 \cdot 593$ & 222643 \\
\hline 43 & 77683 & 1333963 \\
\hline 47 & 1333963 & 1333963 \\
\hline 53,59 & 2404147 & 2404147 \\
\hline 61 & 20950603 & 20950603 \\
\hline 67 & $36254563=127 \cdot 285469$ & 51599563 \\
\hline 71 & 51599563 & 51599563 \\
\hline 73,79 & 96295483 & 96295483 \\
\hline 83 & $114148483=101 \cdot 463 \cdot 2441$ & 146161723 \\
\hline 89 & $269497867=317 \cdot 419 \cdot 2029$ & 1408126003 \\
\hline $97,101,103$ & 269497867 & 3341091163 \\
\hline 107 & $585811843=14081 \cdot 41603$ & 52947440683 \\
\hline 109,113 & 52947440683 & 52947440683 \\
\hline 127 & $71837718283=281 \cdot 3709 \cdot 68927$ & 193310265163 \\
\hline 131,137 & 229565917267 & 229565917267 \\
\hline 139 & $575528148427=149 \cdot 283 \cdot 13648781$ & 915809911867 \\
\hline 149 & 1271259755683 & 1271259755683 \\
\hline $151,157,163$ & 1432817816347 & 1432817816347 \\
\hline
\end{tabular}

It is clear that we are a long way from exhibiting even a single example of $S_{3}(q)>0$ unless its necessary condition is substantially more generous than the sufficient condition (8).

In fact, however, one has [15]

$$
S_{3}(q)=\frac{q^{3} \sqrt{ } q}{\pi}\left[\frac{3}{2 \pi^{2}} L(3, \chi)-L(1, \chi)\right],
$$

where we have corrected an erroneous factor of $\frac{1}{2}$ and where

$$
L(3, \chi)=\prod_{p=2}^{\infty} \frac{p^{3}}{p^{3}-(-q / p)} .
$$

Now

$$
L(3, \chi)>\prod_{p=2}^{\infty} \frac{p^{3}}{p^{3}+1}=\frac{\zeta(6)}{\zeta(3)}=0.84634,
$$

and this gives the sufficient condition (8). In our cases $L(3, \chi)$ will be slightly larger; e.g., for $q=163$, 
TABLE IIIa

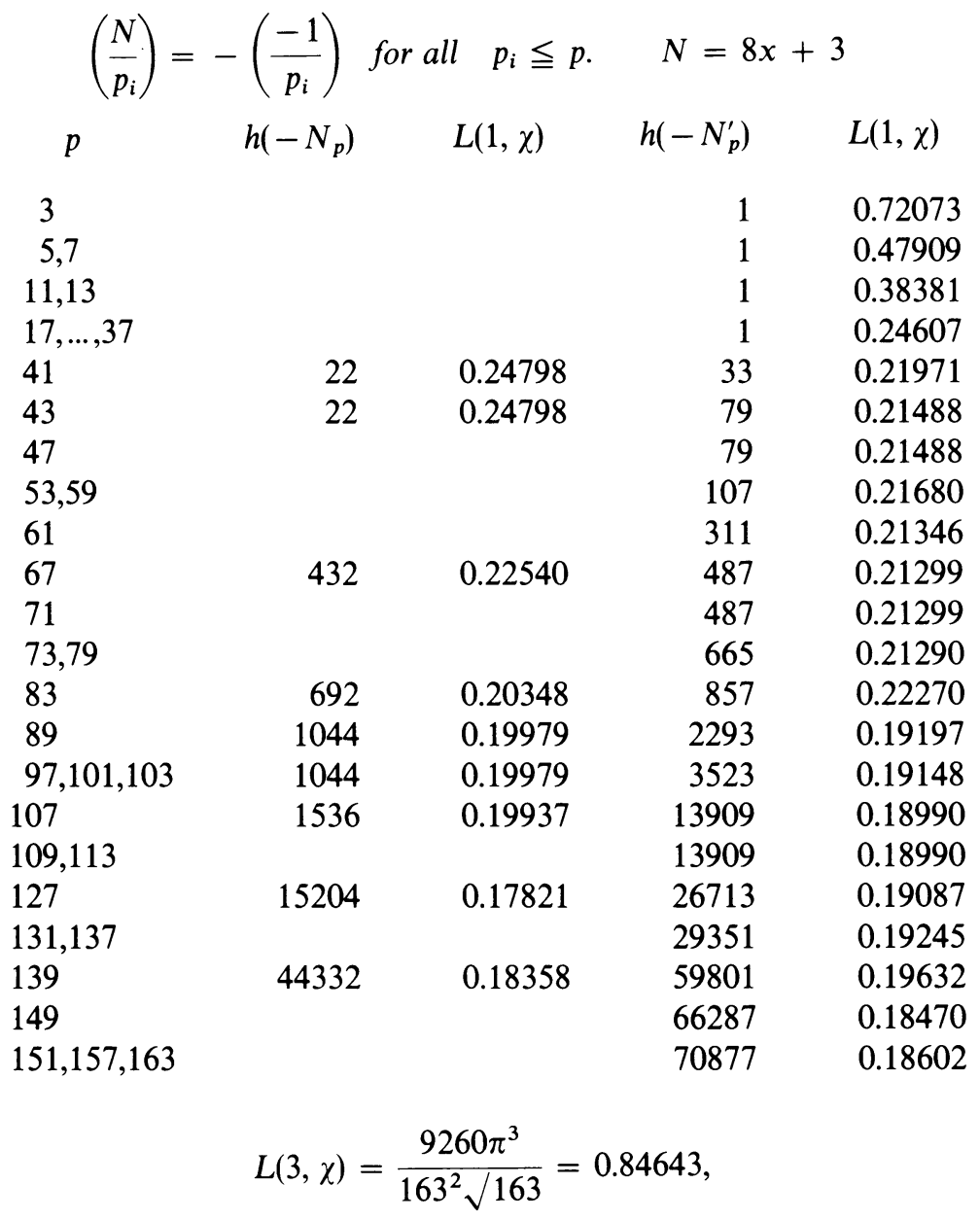

but for $p \geqq 41$ in Table IIIa we must have

$$
L(3, \chi)<\prod_{p=2}^{41} \frac{p^{3}-1}{p^{3}+1} \zeta(3)=0.84644
$$

Therefore,

$$
\frac{3}{2 \pi^{2}} L(3, \chi)<0.12865
$$

and since this is smaller than any $L(1, \chi)$ shown in Table IIIa, we do confirm that $S_{3}(q)<0$ for all of these primes.

In contrast, consider

$$
S_{4}(q)=\sum_{v=1}^{q-1} v^{4}\left(\frac{v}{q}\right)
$$


Now we have

$$
S_{4}(q)=\frac{q^{4} \sqrt{ } q}{\pi}\left[\frac{3}{\pi^{2}} L(3, \chi)-L(1, \chi)\right],
$$

which may be neatly derived from (9) as follows. For any $n \geqq 0$, we obtain

$$
\sum_{v=1}^{q-1} v^{n}(q-v)^{n}\left(\frac{v}{q}\right)=0
$$

since

$$
((q-v) / q)=-(v / q)
$$

and each term $v=a$ cancels that for $v=q-a$.

For $n=2$ and 1 we obtain

$$
S_{4}(q)-2 q S_{3}(q)+q^{2} S_{2}(q)=0, \quad S_{2}(q)-q S_{1}(q)=0,
$$

and thus

$$
S_{4}(q)=2 q S_{3}(q)+q^{4} h(-q) .
$$

Combining (11) and (9) now gives (10).

Therefore, a sufficient condition for $S_{4}(q)>0$ is

$$
L(1, \chi)<\frac{\zeta(6)}{2 \zeta(2) \zeta(3)}=0.25726 .
$$

This condition is met by all $N_{p}^{\prime}$ shown in Table IIIa starting with $N_{37}^{\prime}=163$. (In fact, from (11) and the previously indicated value of $S_{3}(163)$, we have $S_{4}(163)=$ $\left[163^{2}-2(12679)\right] 163^{2}=1211 \cdot 163^{2}$.)

It is reasonable to conjecture that $S_{4}(q)>0$ for all subsequent $N_{p}^{\prime}$ beyond our table, but probably that would be difficult to prove. Presumably, one should attempt to prove it for all $p>p_{0}$ (hopefully small), and then continue the table (if necessary) up to this lower bound $p_{0}$.

In passing, we note that these character sums may be expressed simply in terms of generalized Euler numbers [16]. For $q \equiv 3(\bmod 8)$ we have

$$
\begin{aligned}
& S_{3}(q)=q\left(c_{q, 1}-4 q^{2} c_{q, 0}\right) / 12 \\
& S_{4}(q)=q^{2}\left(c_{q, 1}-2 q^{2} c_{q, 0}\right) / 6 .
\end{aligned}
$$

For example, $c_{163,0}=3$ and $c_{163,1}=166680$, and we may verify the previously indicated sums.

Problem IV. Find $N \equiv-1(\bmod 8)$ as in Problem II, but with $\varepsilon_{i}=-\left(-1 / p_{i}\right)$ as in Problem III. Since the first factor on the right of (7) is now $\frac{2}{1}$ instead of $\frac{2}{3}$ as it was in Problem III, we can expect the values of $h(-N)$ to be about 3 times those of the last problem. It might seem, at first, that these $N$ are of little interest, since we clearly are aiming at small $h(-N)$ and yet we start off immediately in the wrong direction.

But there is another viewpoint. The pre-Kronecker formulation of these problems by Gauss and Dirichlet dealt only with even discriminants. One has the forms 


$$
A u^{2}+2 B u v+C v^{2}
$$

of determinant

$$
D=B^{2}-A C
$$

for every nonsquare integer $D$. The class number is now $h(4 D)$, and the Dirichlet series, for negative $D=-N$ is now

$$
L_{N}(1)=\frac{\pi h(-4 N)}{\sqrt{ } 4 N}=\sum_{k=0}^{\infty}\left(\frac{-N}{2 k+1}\right) \frac{1}{2 k+1}=\prod_{p=3}^{\infty} \frac{p}{p-(-N / p)}
$$

with Jacobi symbol $(-N /(2 k+1))$ and Legendre symbol $(-N / p)$. The quadratic polynomial (6) now becomes

$$
x^{2}+N
$$

and similar questions arise concerning its density of primes, cf. [17]-[18].

It is known that

$$
h(-4 N)=h(-N)
$$

for our present $N \equiv-1(\bmod 8)$, while

$$
h(-4 N)=3 h(-N)
$$

for $N \equiv 3(\bmod 8)$. This nullifies the previously mentioned factor of 3 , and now, using $L_{N}(1)$ instead of $L(1, \chi)$, these two residue classes modulo 8 can be compared on an equal basis, not only with each other, but with any residue class modulo 8 . This gives us a much richer population to study.

We list the first composite and prime solutions in Table IV, while Table IVa gives the values of $h(-N)=h(-4 N)$ and of $L_{N}(1)$. The smallest $L_{N}(1)$ presently known to us is

$$
L_{569078186623}(1)=0.25346 ; \quad(p=137) .
$$

It is smaller than those for any of the $N$ singled out for special mention in the previous problem. These have values

and

$$
\begin{aligned}
& L_{71837718283}(1)=0.26731, \quad(p=127) \\
& L_{85702502803}(1)=0.26172,
\end{aligned}
$$

$$
L_{84148631888752647283}(1)=0.25513 \text {. }
$$

In Problem V below our smallest value is

$$
L_{3666575384938}(1)=0.26064 \quad(p=157) .
$$

Analogous to our remarks concerning $h(-163)$ in the previous problem, we call attention to

$$
h(-4 \cdot 7)=1, \quad h(-4 \cdot 127)=5, \quad h(-4 \cdot 487)=7
$$

in Table IVa. These have been proven [19] to be the largest negative determinants with these class numbers. While the same is probably true of the entry 


$$
\begin{gathered}
\text { TABLE IV } \\
\left(\frac{N}{p_{i}}\right)=-\left(\frac{-1}{p_{i}}\right) \text { for all } p_{i} \leqq p, \quad N=8 x+7
\end{gathered}
$$

\begin{tabular}{|c|c|c|}
\hline$p$ & Least Solution $N_{p}$ & Least Prime Solution \\
\hline 3,5 & 7 & 7 \\
\hline 7 & 127 & 127 \\
\hline 11 & $247=13 \cdot 19$ & 463 \\
\hline 13 & 463 & 463 \\
\hline 17 & 487 & 487 \\
\hline 19 & 1423 & 1423 \\
\hline 23 & 33247 & 33247 \\
\hline 29 & $56743=179 \cdot 317$ & 73327 \\
\hline 31 & $74743=41 \cdot 1823$ & 118903 \\
\hline 37,41 & 118903 & 118903 \\
\hline 43 & $348727=241 \cdot 1447$ & 454183 \\
\hline 47 & 348727 & 773767 \\
\hline $53,59,61$ & 773767 & 773767 \\
\hline 67 & $2430943=227 \cdot 10709$ & 86976583 \\
\hline 71 & 2430943 & 125325127 \\
\hline 73 & 2430943 & 132690343 \\
\hline 79 & $242675623=191 \cdot 263 \cdot 4831$ & 788667223 \\
\hline 83 & $393292183=5573 \cdot 70571$ & 788667223 \\
\hline 89 & 393292183 & 1280222287 \\
\hline 97 & 393292183 & 2430076903 \\
\hline 101 & $1656835783=739 \cdot 827 \cdot 2711$ & 10703135983 \\
\hline 103 & $2713676023=17747 \cdot 152909$ & 10703135983 \\
\hline 107 & $4352137927=64661 \cdot 67307$ & 10703135983 \\
\hline 109 & $8133814327=643 \cdot 12649789$ & 10703135983 \\
\hline 113,127 & 8133814327 & 15605135527 \\
\hline 131 & $8363603623=57047 \cdot 146609$ & 148202808007 \\
\hline 137 & 8363603623 & 569078186623 \\
\hline 139 & $1128864945583=4943 \cdot 228376481$ & 3506439768967 \\
\hline 149,151 & $3402396344407=138727 \cdot 24525841$ & 3506439768967 \\
\hline 157,163 & 3402396344407 & Unknown \\
\hline
\end{tabular}

that remains unproven [19]. But the proposition is not general. For example,

$$
h(-4 \cdot 33247)=53 \text { with } L_{33247}(1)=0.45658
$$

certainly looks unlikely in view of the size of its $L_{N}(1)$, and, in fact, we find in Ordman's table [20] that there is a larger example:

$$
h(-4 \cdot 39103)=53 \text { with } L_{39103}(1)=0.42101 .
$$

It is instructive to note that this latter determinant already fails on $p=13$ : $(-39103 \mid 13)=+1$. 
TABLE IVa

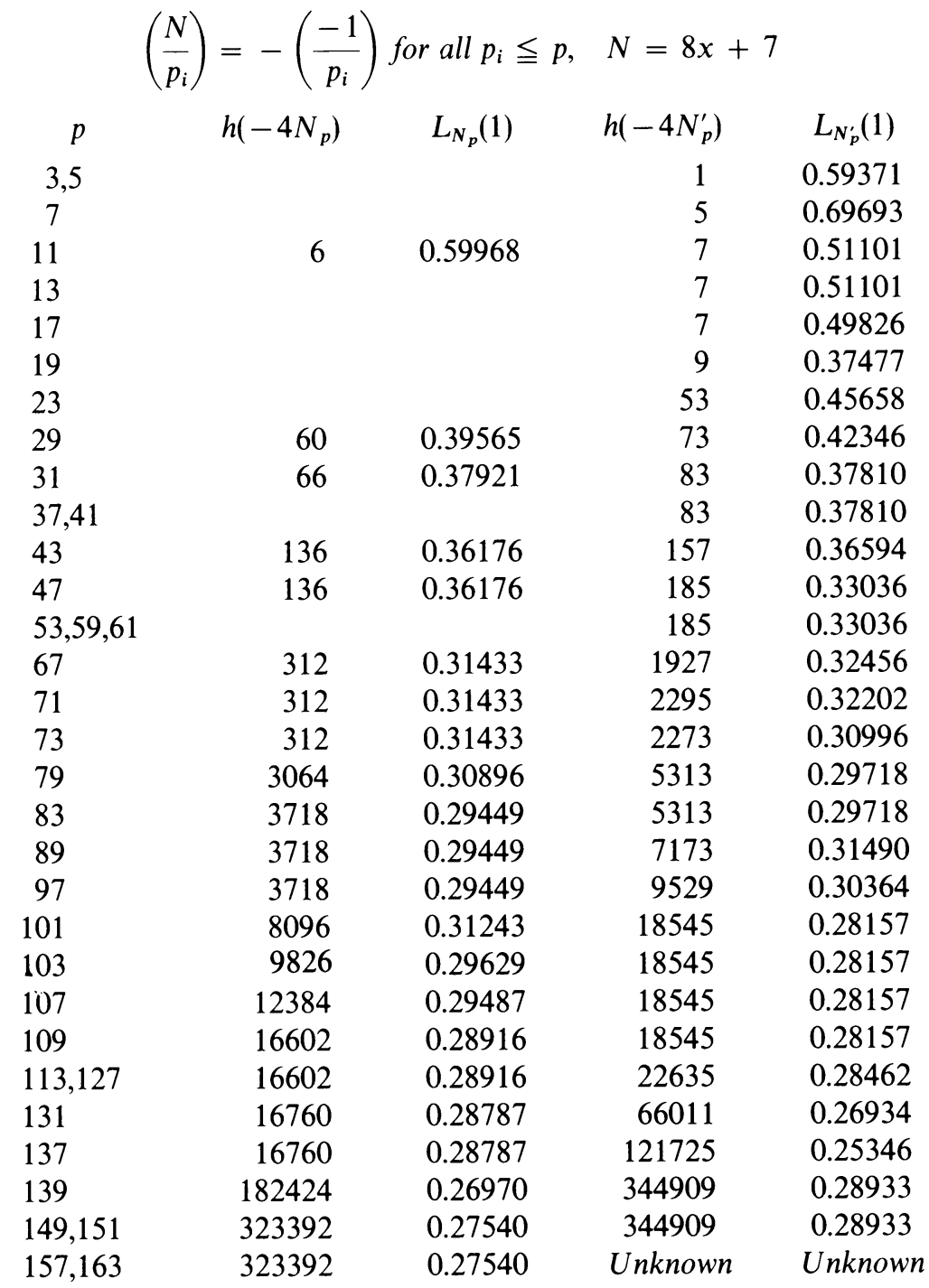

Class Number 3 and a Brief Return to Problem III. The question whether

$$
h(-4 \cdot 1423)=9
$$

exhibits the largest determinant having class number 9 is essentially equivalent to that whether

$$
h(-907)=3
$$

exhibits the largest discriminant having class number 3 . The primes $p=8 x+7$ having $h(-p)=3$ or 9 are completely known [19]:

$$
\begin{gathered}
h(-p)=3 \text { for } p=23,31 \\
h(-p)=9 \quad \text { for } p=199,367,823,1087,1423
\end{gathered}
$$


and if we join these sets, respectively, with all $p=8 x+3$ having $h(-p)=3$, we would obtain the complete set of discriminants with $h(-p)=3$, or the complete set of determinants with $h(-4 p)=9$.

The known $p=8 x+3$ with this class number are [19]:

$$
p=59,83,107,139,211,283,307,331,379,499,547,643,883,907
$$

to which we may add

$$
p^{5}=243
$$

if we do not insist that the discriminant be square-free. There are no other $h(-p)=3$ for $p \leqq 166807$ by the tables of Ordman [20] and Newman [21].

Any $P=8 x+3>1467=3^{2} \cdot 163$ having $h(-P)=3$ must have

$$
L(1, \chi)<0.24607 \text {. }
$$

By a systematic calculation, we find that there are only eight $p=8 x+3<318028$ with an $L(1, \chi)$ that small, and they have these class numbers:

$\begin{array}{cccc}p & h(-p) & p & h(-p) \\ 90787 & 23 & 210907 & 35 \\ 166147 & 29 & 222643 & 33 \\ 191563 & 33 & 253507 & 39 \\ 205627 & 35 & 296587 & 41\end{array}$

Since any further example $P$ must therefore exceed $318028=4 \cdot 43^{3}$, the argument in [19, esp. p. 153, 162], based upon composition of forms, may now be applied as follows. If $(-P \mid q)=+1$, there is a form $F=(q, b, c)$ of discriminant $-P$. Then

\section{TABLE V}

$$
\left(\frac{N}{p_{i}}\right)=-\left(\frac{-2}{p_{i}}\right) \text { for all } p_{i} \leqq p, \quad N=8 x+5
$$

$\quad p$
3
$5, \ldots, 23$
$29, \ldots, 47$
53
59
61,67
71
73
79,83
89,97
$101,103,107$
109
113
$127,131,137$
$139,149,151$
$157, \ldots, 181$

$$
\text { Least Solution } N_{p}
$$$$
5
$$

29

23669

1508789

5025869

$7841261=227 \cdot 34543$

9636461

$18127229=491 \cdot 36919$

$31839341=101 \cdot 239 \cdot 1319$

37989701

240511301

$$
\begin{aligned}
23739440141 & =241 \cdot 367 \cdot 268403 \\
44913466781 & =13339 \cdot 3367079 \\
60664576541 & =149 \cdot 407144809 \\
123464393861 & \\
1833287692469 &
\end{aligned}
$$

Least Prime Solution $N_{p}^{\prime}$

9636461

37989701

37989701

37989701

240511301

41868418349

90664613309

123464393861

123464393861

1833287692469 
$F^{3}=F^{h(-P)}$ represents $q^{3}$ and equals the principal form. Therefore,

$$
q^{3}=u^{2}+u v+(P+1) v^{2} / 4 \text { or } 4 q^{3}=(2 u+v)^{2}+P v^{2} .
$$

Since this is impossible for $4 q^{3}<P$, we must have

$$
(-P / q)=-1
$$

for every $q \leqq 43$. By Tables III and IIIa we therefore have $P>N_{43}^{\prime}=1333963$. But, one also has

$$
4 \cdot 67^{3}<N_{43}^{\prime}, \quad 4 \cdot 163^{3}<N_{67}^{\prime},
$$

and

$$
4 \cdot 7079^{3}<N_{163}^{\prime},
$$

so repetition of the argument shows that

$$
P \geqq N_{7079}^{\prime}>N_{163}^{\prime}=1432817816347,
$$

and

$$
(-P / q)=-1
$$

\begin{tabular}{|c|c|c|c|c|}
\hline \multicolumn{5}{|c|}{ TABLE Va } \\
\hline$\left(\frac{\Lambda}{n}\right.$ & $=-\left(\frac{-2}{n}\right)$ & for all $p_{i} \leqq p$ & $N=8 x+5$ & \\
\hline$p$ & $h\left(-8 N_{p}\right)$ & $L_{2 N_{p}}(1)$ & $h\left(--8 N_{p}^{\prime}\right)$ & $L_{2 N_{p}^{\prime}}(1)$ \\
\hline 3 & & & 2 & 0.99346 \\
\hline $5, \ldots, 23$ & & & 2 & 0.41251 \\
\hline $29, \ldots, 47$ & & & 46 & 0.33210 \\
\hline 53 & & & 406 & 0.36713 \\
\hline 59 & & & 718 & 0.35573 \\
\hline 61,67 & 832 & 0.33002 & 950 & 0.33991 \\
\hline 71 & & & 950 & 0.33991 \\
\hline 73 & 1148 & 0.29949 & 1698 & 0.30599 \\
\hline 79,83 & 1648 & 0.32440 & 1698 & 0.30599 \\
\hline 89,97 & & & 1698 & 0.30599 \\
\hline $101,103,107$ & & & 3990 & 0.28577 \\
\hline 109 & 39880 & 0.28749 & 53510 & 0.29047 \\
\hline 113 & 59012 & 0.30928 & 77970 & 0.28762 \\
\hline $127,131,137$ & 65300 & 0.29448 & 89478 & 0.28285 \\
\hline $139,149,151$ & & & 89478 & 0.28285 \\
\hline $157, \ldots, 181$ & & & 317722 & 0.26064 \\
\hline
\end{tabular}

for all $q \leqq 7079$, are both necessary. Such a $P$ must also have

$$
L(1, \chi)<3 \pi / \sqrt{ } N_{163}^{\prime}=0.0000079,
$$

and therefore either 907 and 1423 are the last examples of $h(-p)=3$ and $h(-4 p)=9$, or any counterexample would (easily) satisfy $S_{3}(P)>0$. We must admit that we would be pleased with either contingency. 
Our next problem is associated with some of the fields having class number 2.

Problem V. Find $N \equiv 5(\bmod 8)$ with $\varepsilon_{i}=-\left(-2 / p_{i}\right)$ for all $p_{i} \leqq p$. This implies that $-2 N$ is a quadratic nonresidue of all odd primes $\leqq p$. The solutions are given in Tables $\mathrm{V}$ and $\mathrm{Va}$, as before, and note, that in this case, $L_{2 N}(1)$ and $L(1, \chi)$ are identical. The first two entries have $h\left(-8 N^{\prime}\right)=2$. Recently, Peter Weinberger [22] proved that $h\left(-8 N^{\prime}\right)$ exceeds 2 for all $N^{\prime}>29$. We also note that, for all $p$,

$$
h\left(-8 N^{\prime}\right) \equiv 2 \quad(\bmod 4) .
$$

This follows from the fact that the only ambiguous form besides the principal form is

$$
\left(2,0, N^{\prime}\right) \text {, }
$$

and this form is not in the principal genus since 2 is a quadratic nonresidue of $N^{\prime}$. Therefore, the class number is a multiple of 2 , but not of 4 .

Relative to Table IV, Table V is quite short since many of its least solutions are valid for a whole string of $p_{i}$, e.g., 123464393861 is valid for six $p_{i}$, and then 1833287692469 is valid for six more. We do not know if this phenomenon is of significance, or merely a fluke. A number of these $N_{p}^{\prime}$ - those for $p=5,29,101$, and 157- have exceptionally small values of $L_{2 N_{p}^{\prime}}(1)$ for determinants of their size.

We round out our choice of quadratic characters by returning to positive discriminants and examining the problem that complements Problem I and extends Problem III into the positive range.

Problem VI. Find $N \equiv 5(\bmod 8)$ with $\varepsilon_{i}=-1$, for all $p_{i} \leqq p$. The least prime residue of $N$ will therefore exceed $p$. The least solutions are given in Table VI. Those for $p=43-53$ were given earlier by N. Beeger and E. Karst [23]. As with Problem I, we have not completed a Table VIa and merely show its beginning:

$\begin{array}{ccccc}p & h\left(N_{p}\right) & L(1, \chi) & h\left(N_{p}^{\prime}\right) & L(1, \chi) \\ 3 & & & 1 & 0.43041 \\ 5 & & & 1 & 0.54002 \\ 7,11 & & & 1 & 0.39091 \\ 13 & & & 1 & 0.33144 \\ 17 & 2 & 0.29106 & 1 & 0.26009 \\ 19,23 & & & 1 & 0.26045 \\ 29 & 4 & 0.25762 & 1 & 0.29195 \\ 31,37,41 & & & 5 & 0.26510\end{array}$

In Table VI we have included 3D values of $L(1, \chi)$ for each $N$. These approximations were obtained by a program called SPEEDY that computes the partial products of (7) for $p<132000$. It evaluates the needed Jacobi symbols by the Reciprocity Law, and requires only a few seconds on an IBM 7094 for each discriminant. While it is very difficult to bound the error of these partial products with a bound that is both realistic and mathematically sound, we know by comparison with many examples where $L(1, \chi)$ is known exactly that usually these SPEEDY approximations are correct to 1 part in 1000 . The very low value of $L(1, \chi)$ for $N_{131}^{\prime}=49107823133$ is of 
TABLE VI

$$
\left(\frac{N}{p_{i}}\right)=-1 \text { for all } p_{i} \leqq p, \quad N=8 x+5
$$

\begin{tabular}{cclll}
$\mu$ & $N_{p}$ & $L(1, \chi)$ & \multicolumn{1}{c}{$N_{p}^{\prime}$} & $L(1, \chi)$ \\
3 & 5 & & 5 & 0.430 \\
5 & 53 & & 53 & 0.540 \\
7,11 & 173 & & 173 & 0.391 \\
13 & 293 & & 293 & 0.331 \\
17 & $437=19 \cdot 23$ & 0.291 & 2477 & 0.260 \\
19,23 & 9173 & 0.258 & 9173 & 0.260 \\
29 & $24653=89 \cdot 277$ & & 74093 & 0.292 \\
$31-41$ & 74093 & & 170957 & 0.265 \\
43 & 170957 & 0.250 & 360293 & 0.246 \\
47 & $214037=193 \cdot 1109$ & 0.250 & 679733 & 0.223 \\
53 & $214037=193 \cdot 1109$ & 0.250 & 2004917 & 0.205 \\
59 & $214037=193 \cdot 1109$ & & 2004917 & 0.205 \\
61 & 2004917 & 0.212 & 69009533 & 0.209 \\
67 & $44401013=157 \cdot 282809$ & 0.226 & 138473837 & 0.233 \\
71 & $94948157=317 \cdot 299521$ & 0.223 & 237536213 & 0.224 \\
73 & $154554077=97 \cdot 1593341$ & 0.223 & 324266477 & 0.227 \\
79 & $154554077=97 \cdot 1593341$ & 0.214 & 324266477 & 0.227 \\
83 & $163520117=2027 \cdot 80671$ & 0.214 & 1728061733 & 0.194 \\
89,97 & $163520117=2027 \cdot 80671$ & 0.190 & 1728061733 & 0.194 \\
101,103 & $261153653=8191 \cdot 31883$ & & 1728061733 & 0.194 \\
$107-113$ & 1728061733 & & 9447241877 & 0.181 \\
127 & 9447241877 & & & \\
131 & $19553206613=14221 \cdot 1374953$ & 0.177 & 49107823133 & $0.169(5)$ \\
137,139 & 49107823133 & & 49107823133 & $0.169(5)$ \\
$149-163$ & $385995595277=191 \cdot 10711 \cdot 188677$ & 0.174 & Unknown & Unknown
\end{tabular}

special interest. It is exceptionally small for a discriminant of this size, and appears to be even smaller than the 0.17009 value mentioned in Problem III.

These Table VI integers $N$ have a pleasing property when considered as negative determinants. All negative determinants $-N$ have ratios

$$
h(-4 N) / \sqrt{ } N
$$

that are asymptotically bounded as $N \rightarrow \infty$ by

$$
A N^{-\varepsilon}<h(-4 N) / \sqrt{ } N<A N^{+\varepsilon}
$$

for any positive $\varepsilon$ [24]. Our present $N$ satisfy

$$
\left(-N / p_{i}\right)=-\left(-1 / p_{i}\right) \quad\left(p_{i} \leqq p\right)
$$

and therefore have

$$
L_{N}(1)=C(p) \prod_{q>p} \frac{q}{q-(-N / q)}
$$


where the coefficient

$$
C(p)=\frac{3}{3-1} \cdot \frac{5}{5+1} \cdot \frac{7}{7-1} \cdot \frac{11}{11-1} \cdot \cdots \cdot \frac{p}{p+(-1 / p)}
$$

converges to $\pi / 2$ as $p \rightarrow \infty$, cf. Euler, Landau [25]. These $N$, therefore, have class numbers $h(-4 N)$ approximately equal to $\sqrt{ } N$ by (14). But the convergence is, of course, quite slow:

$$
\begin{aligned}
& h\left(-4 N_{131}\right)=145644=1.042 \sqrt{ } N_{131}, \\
& h\left(-4 N_{131}^{\prime}\right)=224546=1.013 \sqrt{ } N_{131}^{\prime}, \\
& h\left(-4 N_{149}\right)=592288=0.953 \sqrt{ } N_{149} .
\end{aligned}
$$

There are obviously many similar problems that one can propose and solve with the Delay Line Sieve [2]. The DLS 127 is available to anyone with a suitable problem without charge. We are pleased to acknowledge the assistance of Richard Serafin in computing most of the class numbers.

University of California

Berkeley, California 94720

1180 Miller Avenue

Berkeley, California 94708

Applied Mathematics Laboratory

Naval Ship R\&D Center

Washington, D.C. 20007

1. W. H. MiLls, "Characters with preassigned values," Canad. J. Math., v. 15, 1962, pp. 169-171. MR 28 \# 71.

2. D. H. LeHMER, "An announcement concerning the Delay Line Sieve DLS-127," Math. Comp., v. 20,1966, pp. $645-646$.

3. Marshall Hall, "Quadratic residues in factorization," Bull. Amer. Math. Soc., v. 39, 1933, pp. $758-763$.

4. Allan Cobham, The Recognition Problem for the Set of Perfect Squares, IBM Research Paper, R.C. 1704, April 26, 1966.

5. M. KRAITCHIK, Recherches sur la Théorie des Nombres. Vol. 1, Paris, 1924, pp. 41-46.

6. D. H. LEHMER, "The mechanical combination of linear forms," Amer. Math. Monthly, v. 35, 1928, pp. 114-121.

7. D. H. LeHMER, "A sieve problem on "pseudo-squares"," MTAC, v. 8, 1954, pp. 241-242. MR $16,113$.

8. A. E. Western \& J. C. P. Miller, Indices and Primitive Roots, Royal Soc. Math. Tables, v. 9, Cambridge Univ. Press, New York, 1968, p. xv.

9. Daniel Shanks, "Class number, a theory of factorization, and genera." (To appear.)

10. N. G. W. H. BeEger, "Report on some calculations of prime numbers," Nieuw. Arch. Wiskde, v. 20,1939 , pp. $48-50$. MR 1, 65 .

11. LUIGI PoletTI, "Atlante di centomila numeri primi di ordine quadratico entro cinque miliardi," UMT 62, $M T A C$, v. 2, 1947, p. 354.

12. H. M. STARK, "A complete determination of the complex quadratic fields of class-number one," Michigan Math. J., v. 14, 1967, pp. 1-27. MR 36 \# 5102.

13. D. H. LEHMER, "On the function $X^{2}+X+A$,"Sphinx, v. 6, 1936, pp. 212-214; v. 7, 1937, p. 40; v. 9,1939 , pp. 83-85.

14. Mohan Lal \& Daniel Shanks, "Class numbers and a high density of primes." (To appear.)

15. R. Ayoub, S. Chowla \& H. Walum, "On sums involving quadratic characters," J. London Math. Soc., v. 42, 1967, pp. 152-154. MR 34 \# 4224.

16. Daniel Shanks, "Generalized Euler and class numbers," Math. Comp., v. 21, 1967, pp. 689-694. MR 36 \#6343.

17. Daniel Shanks, "On the conjecture of Hardy \& Littlewood concerning the number of primes of the form $n^{2}+a$," Math. Comp., v. 14, 1960, pp. 320-332. MR 22 \#10960.

18. Daniel Shanks, "Supplementary data and remarks concerning a Hardy-Littlewood conjecture," Math. Comp., v. 17, 1963, pp. 188-193. MR 28 \#3013. 
19. Daniel Shanks, “On Gauss's class number problems,” Math. Comp., v. 23, 1969, pp. $151-163$.

20. EDWARD T. ORDMAN, "Tables of class numbers for negative prime discriminants," UMT 29, Math. Comp., v. 23, 1969, p. 458.

21. MorRIS Newman, "Table of the class number $h(-p)$ for $p$ prime, $p \equiv 3(\bmod 4), 101987 \leqq p \leqq$ 166807," UMT 50, Math. Comp., v. 23, 1969, p. 683.

22. Peter Weinberger, Dissertation, University of California, Berkeley, Calif., June, 1969.

23. EDGAR KARST, "The congruence $2^{p-1} \equiv 1\left(\bmod p^{2}\right)$ and quadratic forms with high density of primes," Elem. Math., v. 22, 1967, pp. 85-88. MR 35 \#6612.

24. C. L. Siegel, "Uber die Classenzahl quadratischer Zahlkorper," Acta Arith., v. 1, 1935, pp. 83-86.

25. E. LANDAU, Handbuch der Lehre von der Verteilung der Primzahlen. Bände 2, Chelsea, New York, 1953, §186, “Euler's Reihen,” pp. 673-676. MR 16, 904. 